Open Access

\title{
An Improvement on the Standard Linear Uncertainty Quantification Using a Least- Squares Method
}

\author{
Heejin Cho ${ }^{1 *}$ D, Rogelio Luck ${ }^{1}$ and James W. Stevens ${ }^{2}$
}

* Correspondence:
cho@me.msstate.edu
${ }^{1}$ Department of Mechanical
Engineering, Mississippi State
University, PO Box 9552, Mississippi
State, MS 39762, USA
Full list of author information is
available at the end of the article

available at the end of the article

\begin{abstract}
Linear uncertainty analysis based on a first order Taylor series expansion, described in ASME PTC (Performance Test Code) 19.1 "Test Uncertainty" and the ISO Guide for the "Expression of Uncertainty in Measurement," has been the most widely technique used both in industry and academia. A common approach in linear uncertainty analysis is to use local derivative information as a measure of the sensitivity needed to calculate the uncertainty percentage contribution (UPC) and uncertainty magnification factors (UMF) due to each independent variable in the measurement/process being examined. The derivative information is typically obtained by either taking the symbolic partial derivative of an analytical expression or the numerical derivative based on central difference techniques. This paper demonstrates that linear multivariable regression is better suited to obtain sensitivity coefficients that are representative of the behavior of the data reduction equations over the region of interest. A main advantage of the proposed approach is the possibility of extending the range, within a fixed tolerance level, for which the linear approximation technique is valid. Three practical examples are presented in this paper to demonstrate the effectiveness of the proposed least-squares method.
\end{abstract}

Keywords: Uncertainty, Sensitivity analysis, Linear regression, Covariance

\section{Springer}

\section{Introduction}

The topic of estimation of experimental uncertainty is covered in a wide variety of forums. The American Society of Mechanical Engineers publishes an uncertainty standard as part of the performance test codes: ASME PTC 19.1-1998 "Test Uncertainty" [1]. The International Organization for Standardization (ISO) also publishes a guide on uncertainty calculation and terminology entitled "Guide to the Expression of Uncertainty in Measurement" [2]. These two approaches are compared by Steele et al. [3]. Most textbooks on experimental measurements include a section on uncertainty propagation as well (for example, Refs. [4-6]). Some textbooks specialize in uncertainty [7, 8]. The technical literature also has numerous treatments of uncertainty estimation and propagation in specific applications (for example, Refs. [9-12]). Although there are more sophisticated uncertainty quantification methods, including Monte Carlo [13], Bayesian [14], Latin square sampling techniques [15, 16], by far ASME PTC 19.1-1998 "Test Uncertainty" standard [1] is the most widely adopted in the current industrial applications. A main goal of this paper is to provide a simple improvement to the practical method provided by the ASME standard. 
One element of experimental uncertainty analysis deals with the manner in which uncertainty in measurements propagates into a final result. The validity of the uncertainty estimate of the result rests on both the validity of the measurement uncertainties and the method of propagation of those uncertainties through the analysis equation. A 2-D example of the uncertainty analysis concept is presented in Fig. 1. Point $\left(x^{*}, y^{*}\right)$ has a probability, $\mathrm{P}^{*}$, obtained from the probability density function, $\operatorname{pdf}_{\mathrm{xy}}$, i.e., $P^{* *}=\operatorname{pdf}_{\mathrm{xy}}\left(\mathrm{x}^{*}, \mathrm{y}^{*}\right)$. This point is evaluated through the function of $f$ to find a value of $z^{*}=\mathrm{f}\left(\mathrm{x}^{*}, \mathrm{y}^{*}\right)$ and the probability, $P_{\mathrm{z}}^{*}$, of $z^{*}$ is obtained from $\operatorname{pdf}_{\mathrm{z}}\left(z^{*}\right)$. The objective is to find the confidence interval (95\% is commonly used in engineering applications) for the output of $z$.

Most approaches [Coleman and Steele [7], Abernethy et al. [17], International Organization for Standardization (ISO) Guide [2], etc. to engineering uncertainty propagation are based on the assumption of linear behavior for small perturbations in the measured variables. These approaches rely on a first order Taylor series approximation at a nominal location obtained from the mean of the measured variables. The problem with the first order Taylor series approximation arises when large truncation errors are present. While the Taylor series approximation is usually quite good over regions of high probability, it can give a very poor estimate of the $95 \%$ confidence interval for highly non-linear functions. This is illustrated conceptually in Fig. 2. In this paper, the authors describe a least-squares approach to obtain better sensitivity coefficients that result in better predictions for the $95 \%$ confidence interval.

\section{Background}

\section{Total Uncertainty and Covariance Matrix}

For Gaussian distributed variables, the total uncertainty can be obtained by summing the square of bias and precision uncertainties [7];

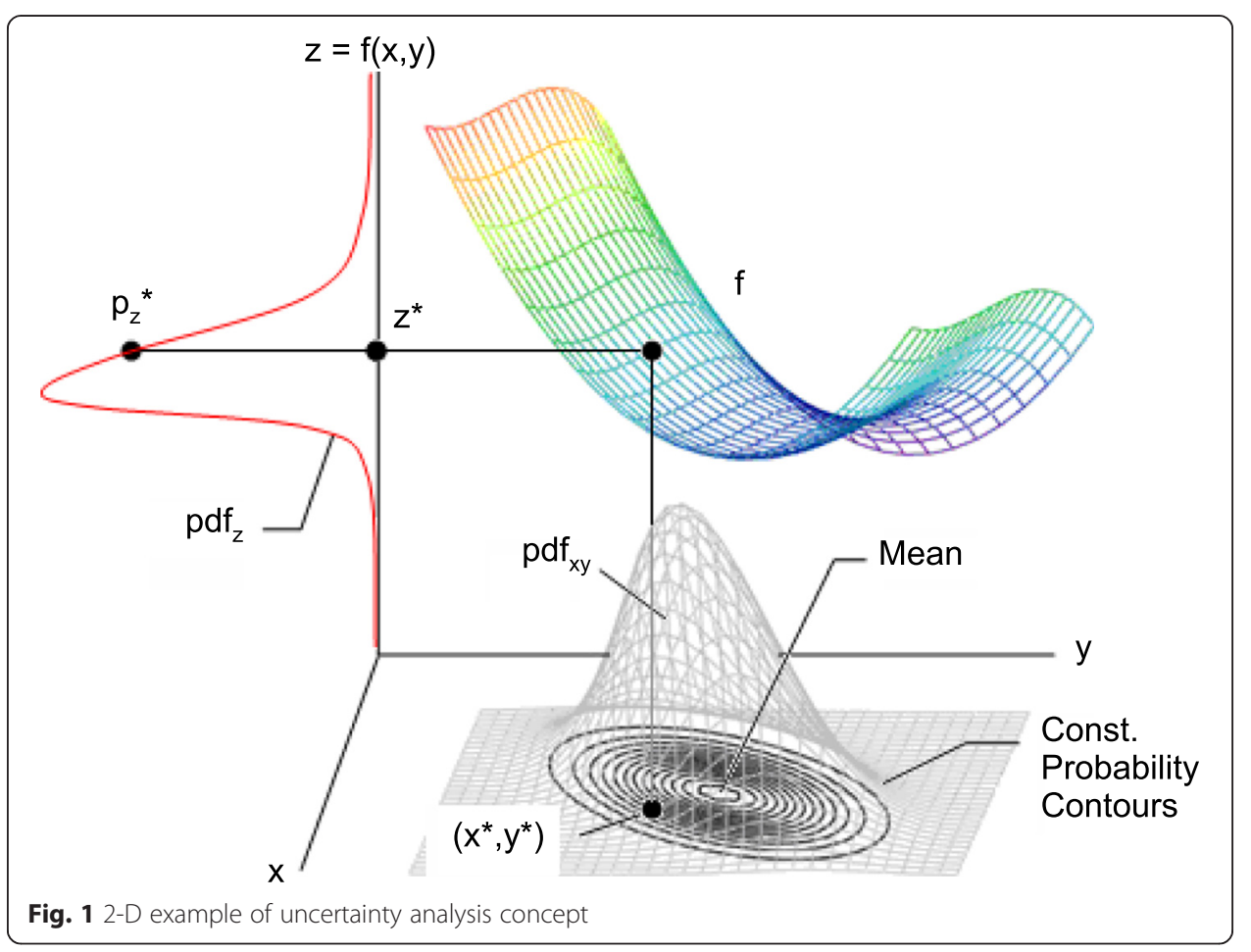




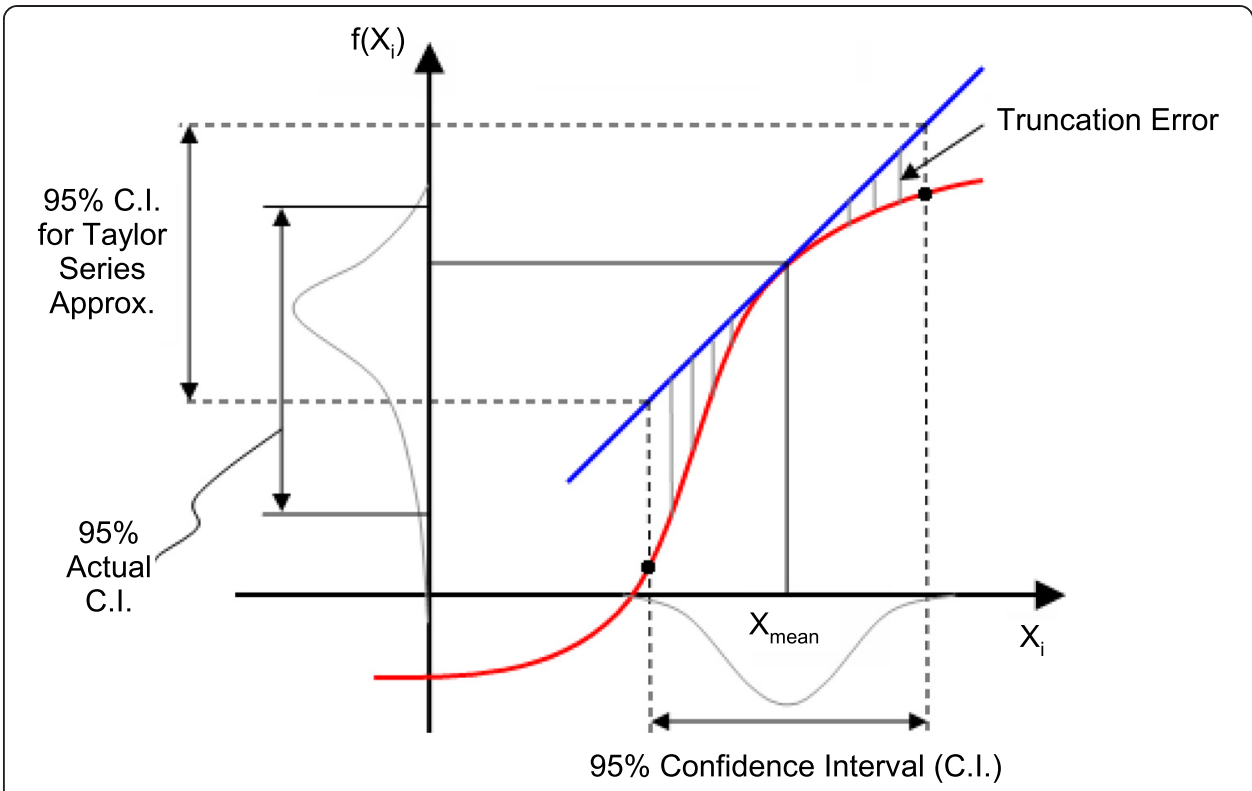

Fig. 2 Truncation errors by Taylor series approximation at a nominal point within uncertainty region

$$
U_{T}^{2}=U_{B}^{2}+U_{P}^{2}
$$

where $U_{\mathrm{T}}, U_{\mathrm{B}}$, and $U_{\mathrm{P}}$ are the total, bias, and precision uncertainty, respectively. The bias and precision uncertainties are obtained separately at a prescribed confidence interval (i.e., at $95 \%$ confidence interval). The total bias and precision uncertainties are then calculated using the sensitivity coefficient (gradient) of each variable in the analysis equation. The total bias uncertainty is obtained from: [7]

$$
U_{B}^{2}=\sum_{i=1}^{J}\left(\theta_{i} \cdot B_{i}\right)^{2}+2 \sum_{i=1}^{J-1} \sum_{k=i+1}^{J} \theta_{i} \cdot \theta_{k} \cdot B_{i k}
$$

where $\theta_{\mathrm{i}}$ is the gradient $\left(\frac{d f\left(X_{i}\right)}{d X_{i}}\right)$ of each variable, $B_{\mathrm{i}}$ is the bias uncertainty of each variable, and $B_{\mathrm{ik}}$ is the correlation among variables. The bias uncertainties of each variable are generally given by the manufacturer or are estimated by engineering judgment. Bias uncertainties of some variables may be correlated, e.g., when two thermistors are calibrated by the same thermometer, they will share a common error due to the uncertainties in the thermometer. The total precision uncertainty is obtained from: [7]

$$
U_{P}^{2}=\sum_{i=1}^{J}\left(\theta_{i} \cdot P_{i}\right)^{2}
$$

where $P_{\mathrm{i}}$ is the precision uncertainty of each variable. Precision uncertainties represent the random scattering of each variable and are assumed to be uncorrelated.

Alternatively, the total uncertainty can be determined using covariance matrices. For a Gaussian distribution, covariance matrices of the bias and precision propagations for 2-D case can be determined as 


$$
\begin{aligned}
& C_{\text {Bias }}=\left[\begin{array}{cc}
\sigma_{X, B}{ }^{2} & \rho \cdot \sigma_{X, B} \cdot \sigma_{Y, B} \\
\rho \cdot \sigma_{X, B} \cdot \sigma_{Y, B} & \sigma_{Y, B}{ }^{2}
\end{array}\right] \\
& C_{\text {Precision }}=\left[\begin{array}{cc}
\sigma_{X, P}{ }^{2} & 0 \\
0 & \sigma_{Y, P}{ }^{2}
\end{array}\right]
\end{aligned}
$$

where $\rho$ is the correlation coefficient of the bias errors and $\sigma$ is the standard deviation. Guidelines for bias errors and correlation coefficients for realistic experimental situations are given by Coleman and Steele [7]. Since bias and precision errors are statistically independent, their covariance matrices can be added to obtain the covariance matrix of the combined error.

$$
C=\left[\begin{array}{cc}
\sigma_{X, B}^{2}+\sigma_{X, P}^{2} & \rho \cdot \sigma_{X, B} \cdot \sigma_{Y, B} \\
\rho \cdot \sigma_{X, B} \cdot \sigma_{Y, B} & \sigma_{Y, B}^{2}+\sigma_{Y, P}^{2}
\end{array}\right]
$$

The total standard deviation is

$$
\sigma_{T}=\sqrt{B^{T} \cdot C \cdot B}
$$

where $A$ is an nx1 matrix which contains sensitivity coefficients of each variable, e.g., $B=\left[\theta_{1} \theta_{2}\right]^{\mathrm{T}}$, and $C$ is an nxn covariance matrix (Eq. (6)) and is assumed symmetric and positive definite. The total uncertainty is then determined as

$$
U_{T}=t \cdot \sigma_{T}
$$

where $t$ is the value obtained from the t-distribution corresponding to a particular confidence level (i.e., $95 \%$ confidence level corresponds to $1.96 t$ value) [2].

\section{Covariance Matrix Based on Uncorrelated Bias Errors}

Consider a scalar function $f(\bar{x})$ of $n$ variables. The errors, $\bar{e}$, can be expressed in terms of sources of error that are assumed to be uncorrelated. In such a case, the covariance matrix can be obtained as follows:First the errors in $\bar{x}$ are expressed as:

$$
\bar{e}=\left[\begin{array}{ll}
I & A
\end{array}\right] \cdot\left[\begin{array}{c}
\bar{e}_{p} \\
\bar{e}_{b}
\end{array}\right]
$$

where $\bar{e}_{p}$ are the precision errors, $\bar{e}_{b}$ are the bias errors associated with $\bar{x}, I$ is the nxn identity matrix and $A$ is an nxm matrix where $m$ is the number of uncorrelated bias terms. Matrix $A$ is made up of zeros and ones and determines how each source of bias error influences a particular input. The covariance matrix is then given by:

$$
\begin{aligned}
& C=E\left(\bar{e} \bar{e}^{T}\right)=\left[\begin{array}{ll}
I & A
\end{array}\right] \cdot E\left(\left[\begin{array}{cc}
\bar{e}_{p} \bar{e}_{p}^{T} & \bar{e}_{p} \bar{e}_{b}^{T} \\
\bar{e}_{b} \bar{e}_{p}^{T} & \bar{e}_{b} \bar{e}_{b}^{T}
\end{array}\right]\right) \cdot\left[\begin{array}{c}
I \\
A^{T}
\end{array}\right]
\end{aligned}
$$

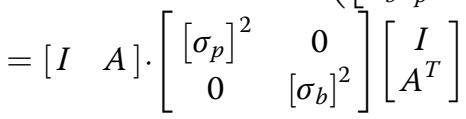

or

$$
C=\left[\sigma_{p}\right]^{2}+A \cdot\left[\sigma_{b}\right]^{2} \cdot A^{T}
$$

where $\left[\sigma_{p}\right]^{2}$ and $\left[\sigma_{b}\right]^{2}$ are diagonal nxn and mxm matrices, respectively. In the example section, Eq. (10) is used to estimate the $95 \%$ of confidence interval of a convective heat transfer coefficient $(h)$. 


\section{Gaussian multivariate probability density function}

The Gaussian multivariate probability density function (pdf) in two dimensions is given by

$$
\operatorname{pdf}(x, y)=\frac{e^{-\frac{1}{2} \cdot\left[\begin{array}{l}
x \\
y
\end{array}\right]^{T} \cdot C^{-1} \cdot\left[\begin{array}{l}
x \\
y
\end{array}\right]}}{\sqrt{(2 \pi)^{2} \cdot|C|}}
$$

A 3-D plot of this $\operatorname{pdf}(x, y)$ is shown in Fig. 3. For dimensions larger than two, Eq. (11) can be expanded to account for the additional dimensions.

\section{Development}

\section{Geometrical Interpretations}

Covariance matrices can be decomposed as [18]

$$
C=S \cdot \Lambda \cdot S^{T}
$$

where $S$ is an orthonormal matrix (rotation matrix) containing a set of orthonormal eigenvectors of $C$, and $\Lambda$ is a diagonal matrix containing the eigenvalues of $C . \sqrt{\Lambda_{0,0}}$ and $\sqrt{\Lambda_{1,1}}$ represent the principal standard deviations about the rotated coordinates, e.g., the $x$ and $y$ coordinate system shown in the 3-D example in Fig. 3. Using the tdistribution, major and minor axes for an ellipse that bounds the $95 \%$ probability region in 2-D are obtained by multiplying $\sqrt{\Lambda_{0,0}}$ and $\sqrt{\Lambda_{1,1}}$ by $t=1.96$. The resulting ellipse can then be divided into small sections. One way to partition the sections is to ensure that each section has the same probability. Another way to create sections is to ensure that each section has the same area.

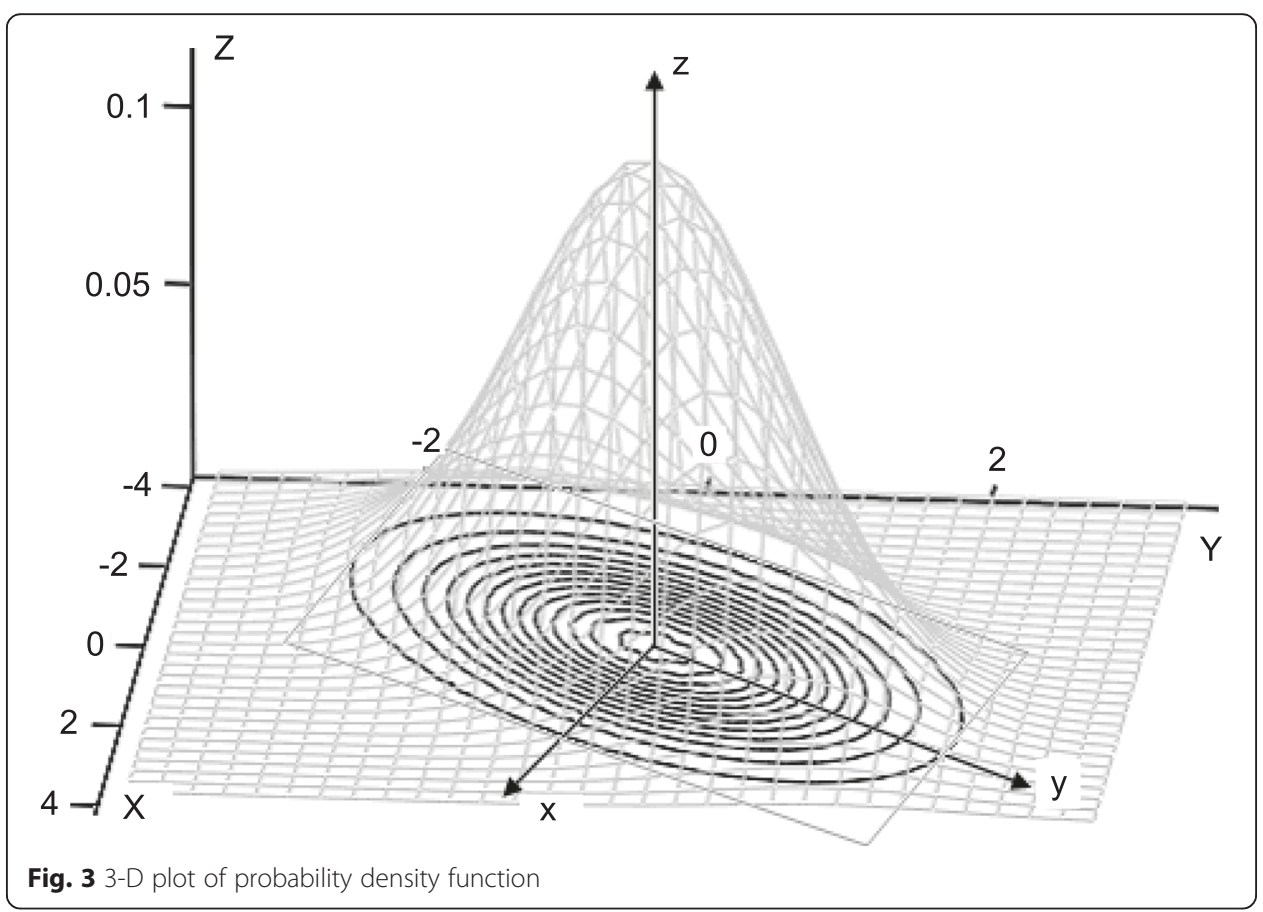


Roberts et al. [19] suggest that the uniform space approach requires fewer function evaluations compared to the uniform probability approach to obtain comparable results for 1-D cases. Therefore, uniform-space geometry is used to describe the uncertainty region in this paper. An easy way to generate a uniform-space grid is to inscribe the elliptical area within a rectangular area. Furthermore, to generate a large number of samples for an increased accuracy of the uncertainty results, an efficient sampling technique, such as Latin hypercube sampling technique $[15,16]$, can be employed to reduce computational cost.

\section{Least-Squares Approach}

The first order Taylor series approximation at a nominal point in the measured variables is often used to determine engineering uncertainty. However, this approach fails when applied to systems that are nonlinear in the uncertainty region so that the large truncation errors occur. A least-squares approximation minimizes the truncation errors. Therefore, the least-squares approximation can provide better representation of the analysis equation, especially in the region of the confidence interval, as shown in Fig. 4.

Figure 4 shows that the least-squares approach yields larger truncation errors near the high probability region, but improves the truncation errors over the entire the interval of interest leading to a better estimate of the $95 \%$ confidence interval. Although Fig. 4 illustrates a conceptual comparison, the results of the examples represented in the following section support this idea.

For a two dimensional problem, the data reduction function is fit with a plane. The 2-D least-square plane can be obtained as follows.

$$
\bar{f}(x, y)=a \cdot x+b \cdot y+c
$$

where $a, b$, and $c$ are the least-square coefficients.

The uncertainty values can be obtained by applying Eqs. (1), (2), and (3) using the sensitivity coefficients based on the least-squares approximation:

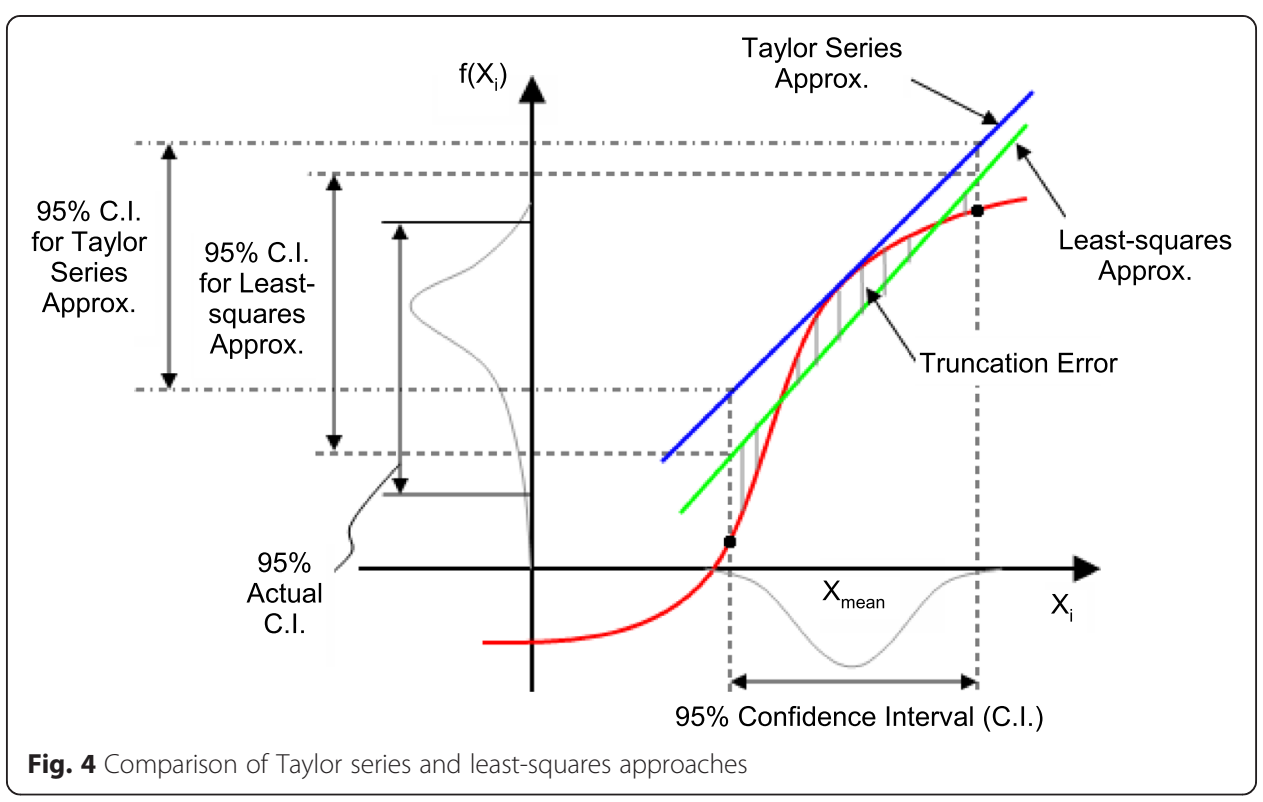




$$
\theta_{\mathrm{i}}=\frac{d \bar{f}\left(X_{i}\right)}{d X_{i}}
$$

Note that the sensitivity coefficients in Eq. (14) are equal to the values of $a$ and $b$ from Eq. (13). Alternatively, Eqs. (7) and (8) can be used to find the uncertainty bands which must be centered about the mean of the least-squares approximation. This latter mean is given by

$$
\bar{f}\left(\mu_{x}, \mu_{y}\right)=\left(c+a \cdot \mu_{x}+b \cdot \mu_{y}\right)
$$

\section{Piece-wise approach}

The piece-wise approach is a method used to estimate probability distribution by sorting discrete probabilities cumulatively. This method was described in detail for one dimension in [20]. The extension to multiple dimensions can be used to estimate accurate values for the confidence interval in cases where the exact solution is not available.

\section{Examples}

Three simple examples will be presented to illustrate the least-squares approach to uncertainty estimation.

The first example uses a simple one-dimensional parabola as the data reduction equation. This equation has the benefits of extreme simplicity and a readily accessible exact result for calculating the confidence interval. The equation is:

$$
y=x^{2}+0.5
$$

The asymmetric $90 \%$ confidence interval was calculated over a range of values in the independent variable, $x$, using 20 points for the least-square fit. In all cases, the leastsquare result was closer to the exact solution for the upper and lower bounds of the $90 \%$ confidence interval than the Taylor series estimate. Figure 5 shows the results of the calculations between $1<\mathrm{x}<2.5$.

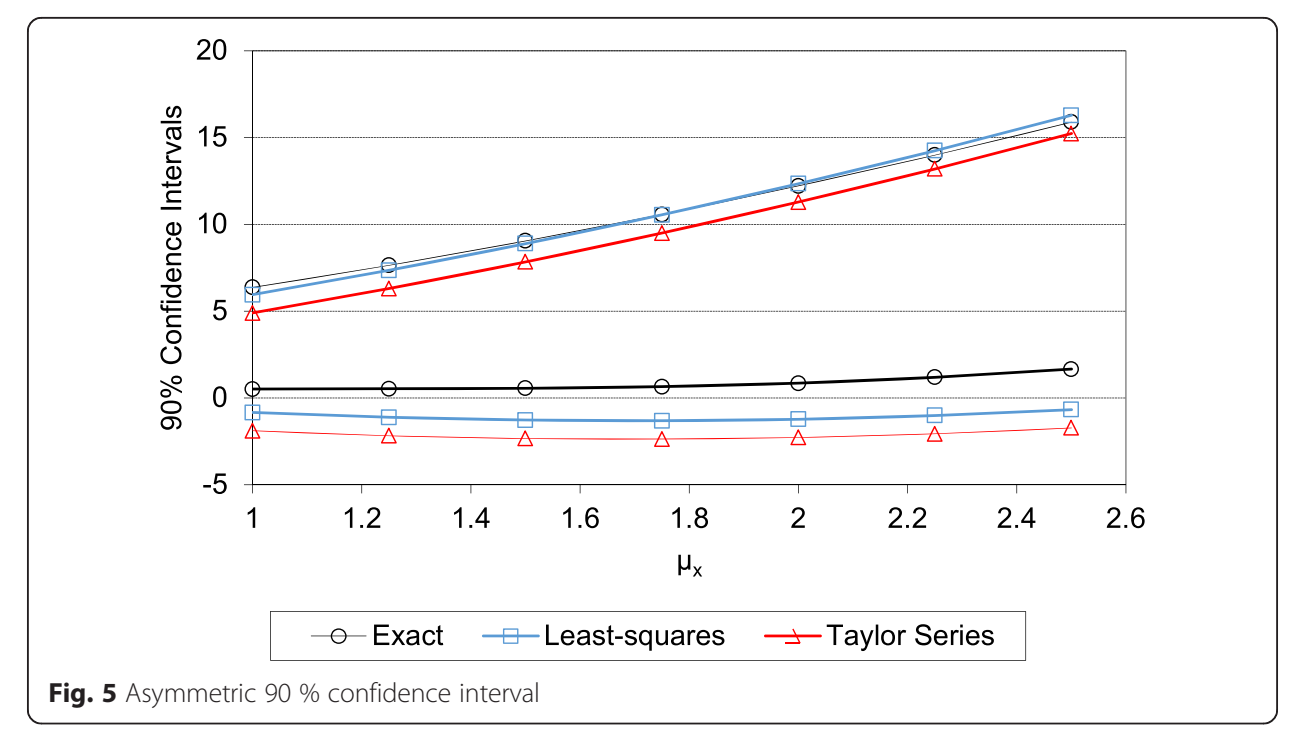




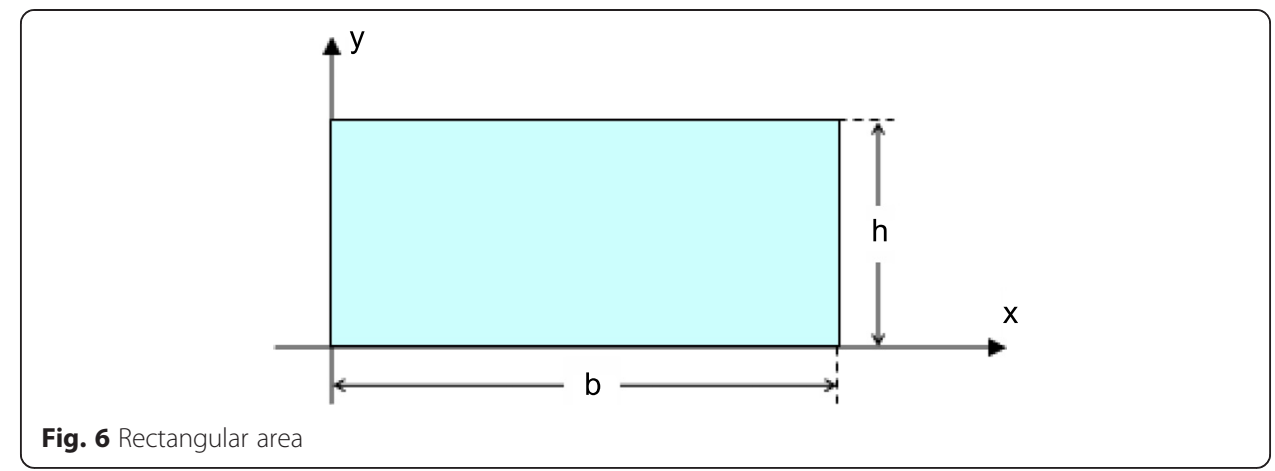

The second example uses a moment of inertia calculation for the rectangular area shown in Fig. 6. It is desired to find the moment of inertia about the $\mathrm{x}$ axis along with the associated uncertainty. The analysis function is:

$$
I=\frac{b \cdot h^{3}}{3}
$$

where $b$ and $h$ are the base and height lengths of the rectangular. Assume that a ruler is used to measure the lengths of $b$ and $h$. For this example, the nominal values of $b$ and $h$ are measured as 5 and $3 \mathrm{~cm}$, respectively. Since both measurements of $b$ and $h$ are measured from the same device, the values are correlated. The bias uncertainty of the ruler is given by $0.5 \mathrm{~cm}$ from the manufacturer. The correlation in the bias, $\rho$, is 1 since the same ruler is used to measure $b$ and $h$. We assume that bias uncertainty has $95 \%$ confidence level, so the standard deviation for bias uncertainty is $0.255 \mathrm{~cm}(=0.5 / 1.96)$. Suppose that 20 measurements each of $b$ and $h$ are taken in order to find precision uncertainty. Standard deviations of each variable $b$ and $h$ are calculated as 0.2 and $0.3 \mathrm{~cm}$, respectively, based on these measurements. These values are summarized in Table 1.

Bias and precision covariance matrices are determined by Eqs. (4) and (5).

$$
C_{B}=\left[\begin{array}{cc}
0.255^{2} & (1) \cdot(0.255) \cdot(0.255) \\
(1) \cdot(0.255) \cdot(0.255) & 0.255^{2}
\end{array}\right] \text { and } C_{P}=\left(\begin{array}{cc}
0.2^{2} & 0 \\
0 & 0.3^{2}
\end{array}\right)
$$

The covariance matrix, linearly combined by $C_{\mathrm{B}}$ and $C_{\mathrm{B}}$ is then defined by Eq. (6).

$$
C=\left(\begin{array}{ll}
0.105 & 0.065 \\
0.065 & 0.155
\end{array}\right)
$$

Table 1 Numerical values of parameters used to find uncertainty

\begin{tabular}{lll}
\hline & $b$ (Base) & (Unit: cm) \\
\hline Mean (nominal value) & 5 & $h$ (Height) \\
Bias uncertainty & 0.5 & 3 \\
Bias standard deviation & $0.255(=0.5 / 1.96)$ & \\
Correlation in bias & 1 & 0.3 \\
Precision standard deviation & 0.2 & \\
\hline
\end{tabular}




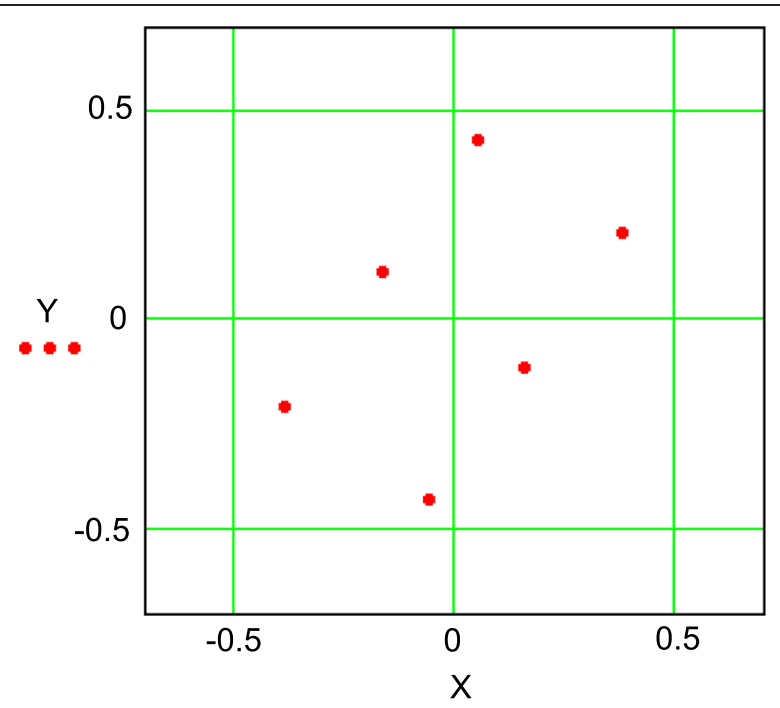

Fig. 7 Results from geometrical interpretation in Section 3.1 for least-squares approach

The matrices $\Lambda$ and $S$ are then determined from eigenvalue decomposition of the covariance matrix: $\Lambda=\left[\begin{array}{cc}0.06 & 0 \\ 0 & 0.2\end{array}\right]$ and $S=\left[\begin{array}{cc}0.824 & 0.566 \\ -0.566 & 0.824\end{array}\right]$

A set of six points shown in Fig. 7 is sampled based on the uniform space approach [19] as discussed in Section 3.1 and these points represent an uncertainty region for Eq. (17). The points shown in Fig. 7 are evaluated using the original function, Eq. (17), and its result is used to perform a least-squares fit to determine the equation of the least-squares plane, Eq. (13). The result of this fit is shown below.

$\bar{f}(x, y)=9.20 \cdot x+47.2 \cdot y+54.4$

where $x$ and $y$ represent the deviation from the nominal values of $b$ and $h$.

The total uncertainty at $95 \%$ confidence level is obtained using Eqs. (7) and (8) to obtain

$$
U_{\mathrm{T}}= \pm 39.8\left(\mathrm{~cm}^{4}\right)
$$

The mean of the least-squares fit is given by

$$
\bar{f}\left(\mu_{x}, \mu_{y}\right)=54.4\left(\mathrm{~cm}^{4}\right)
$$

The $95 \%$ confidence region is then given by

$$
14.6 \leq f(x, y) \leq 94.2\left(\mathrm{~cm}^{4}\right)
$$

Finally, the nominal value is obtained from the original function:

$$
f\left(\mu_{x}, \mu_{y}\right)=45\left(\mathrm{~cm}^{4}\right)
$$

and it is seen that the $95 \%$ confidence bounds are not symmetrical about the nominal value from the original function. 


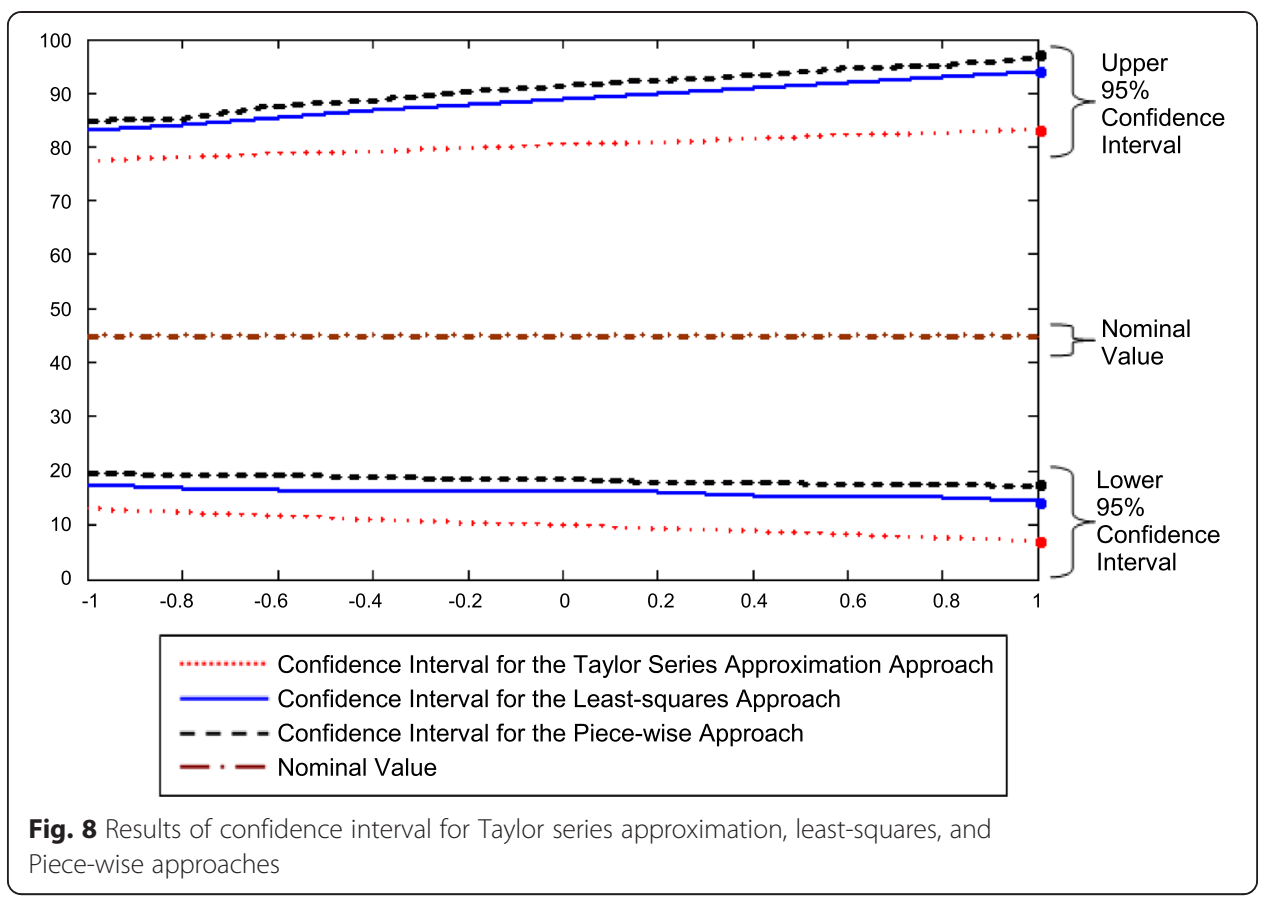

The results of the above method are compared to a more accurate solution obtained by the piece-wise approach which sections the input XY probability region into a thousand points.

The results of $95 \%$ confidence interval for Taylor series approximation, least-squares, and piece-wise approaches are compared in Fig. 8. The results are compared as the correlation coefficient, $\rho$, changes from -1 to 1 . Figure 8 shows that the confidence interval for the least-squares approach provides a better approximation to the more realistic (but much more computationally intensive) piece-wise approach than does the Taylor series approach.

The third example demonstrates application of the least-squares approach to a 3dimensional problem. A gas temperature measurement system is used for this example. A thermocouple is used to measure the exhaust gas temperature of a diesel engine. The thermocouple is $3.175 \mathrm{~mm}$ (1/8 in.) diameter. The system is illustrated in Fig. 9. Assuming the system is at steady-state and that the thermocouple and wall are both at

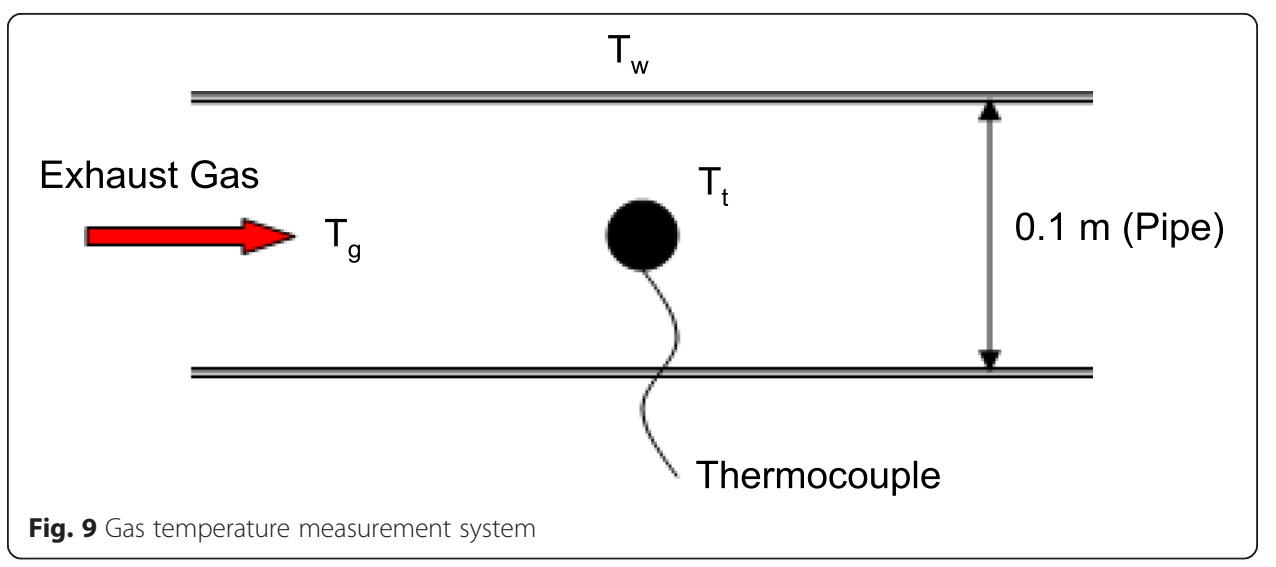


uniform temperature and neglecting heat conduction through the thermocouple wires, the equation used to determine the gas temperature is

$$
h \cdot\left(T_{g}-T_{t}\right)=\varepsilon \cdot \sigma \cdot\left(T_{t}^{4}-T_{w}^{4}\right)
$$

(convection to the probe $=$ radiation from the probe $)$

where

$T_{\mathrm{g}}$ gas temperature (K)

$T_{\mathrm{t}}$ thermocouple temperature (K)

$T_{\mathrm{w}}$ pipe-wall temperature (K)

$\varepsilon$ emissivity of the thermocouple

$\sigma$ Stefan-Boltzmann constant $\left(2.043 \times 10^{-7} \mathrm{~kJ} / \mathrm{hr}-\mathrm{m}^{2}-\mathrm{K}^{4}\right)$

$h$ convective heat transfer coefficient $\left(\mathrm{kJ} / \mathrm{hr}-\mathrm{m}^{2}-\mathrm{K}\right)$.

It is desired to investigate whether it is a good idea to use Eq. (18) to determine the convective heat transfer coefficient. To this effect, Eq. (18) can be rewritten as

$$
h=\frac{\varepsilon \cdot \sigma \cdot\left(T_{t}^{4}-T_{w}^{4}\right)}{\left(T_{g}-T_{t}\right)}
$$

Assume that a thermocouple calibration block which introduces an error with standard deviation of $3 \mathrm{~K}$ is used to calibrate both the thermocouple shown in Fig. 9 and a thermocouple used to measure the temperature of the pipe-wall. It follows that these thermocouples are correlated, and the common bias error is found to have a standard deviation of $3 \mathrm{~K}$. Assume that a thermometer is used to measure the gas temperature and the standard deviation of the bias is given from the manufacturer as $4 \mathrm{~K}$. The nominal, bias, and precision values of each variable are listed in Table 2.

The precision and bias standard deviations shown in Table 2 are used to determine the covariance matrix in Eq. (10). The covariance is then obtained as

$$
\begin{aligned}
C & =\left[\begin{array}{ll}
I & A
\end{array}\right] \cdot\left[\begin{array}{cc}
{\left[\sigma_{p}\right]^{2}} & 0 \\
0 & {\left[\sigma_{b}\right]^{2}}
\end{array}\right] \cdot\left[\begin{array}{c}
I \\
A
\end{array}\right] \\
& =\left[\begin{array}{lllll}
1 & 0 & 0 & 1 & 0 \\
0 & 1 & 0 & 1 & 0 \\
0 & 0 & 1 & 0 & 0
\end{array}\right] \cdot\left[\begin{array}{ccccc}
12^{2} & 0 & 0 & 0 & 0 \\
0 & 10^{2} & 0 & 0 & 0 \\
0 & 0 & 14^{2} & 0 & 0 \\
0 & 0 & 0 & 6^{2} & 0 \\
0 & 0 & 0 & 0 & 8^{2}
\end{array}\right] \cdot\left[\begin{array}{lll}
1 & 0 & 0 \\
0 & 1 & 0 \\
0 & 0 & 1 \\
1 & 1 & 0 \\
0 & 0 & 1
\end{array}\right]
\end{aligned}
$$

Note that correlation of the variables can be determined in the matrix $\left[\begin{array}{ll}I & A\end{array}\right]$.

The same procedure presented in the previous example is applied to this problem after obtaining the covariance matrix. The result of the least-squares fit is determined as

Table 2 Nominal, bias, and precision values of each variable

\begin{tabular}{llllll}
\hline & $\begin{array}{l}T_{g} \\
\text { (Temp. gas) }\end{array}$ & $\begin{array}{l}T_{\mathrm{t}} \text { (Temp. } \\
\text { thermocouple) }\end{array}$ & $\begin{array}{l}T_{\mathrm{w}} \text { (Temp. } \\
\text { pipe-wall) }\end{array}$ & $\varepsilon$ (Emissivity) & $\sigma$ (Stefan-Boltzmann constant) \\
\hline $\begin{array}{l}\text { Mean (nominal value) } \\
\begin{array}{l}\text { Precision standard } \\
\text { deviation }\end{array}\end{array}$ & $838 \mathrm{~K}$ & $811 \mathrm{~K}$ & $672 \mathrm{~K}$ & 0.55 & $2.043 \times 10^{-7} \mathrm{k} / \mathrm{hr}^{-\mathrm{m}^{2}-\mathrm{K}^{4}}$ \\
$\begin{array}{l}\text { Bias standard } \\
\text { deviation }\end{array}$ & $4 \mathrm{~K}$ & $3 \mathrm{~K}$ & $5 \mathrm{~K}$ & $\mathrm{~N} / \mathrm{A}$ & $\mathrm{N} / \mathrm{A}$ \\
\hline
\end{tabular}


$\bar{f}(x, y, z)=99.9 \cdot x-61.4 \cdot y-88.4 \cdot z-975$

The total uncertainty at $95 \%$ confidence level is obtained using Eqs. (7) and (8) to obtain

$$
U_{\mathrm{T}}= \pm 2.28 \times 10^{3}\left(\mathrm{~kJ} / \mathrm{hr}-\mathrm{m}^{2}-\mathrm{K}\right)
$$

The mean of the least-squares fit is given by

$$
\bar{f}\left(\mu_{x}, \mu_{y}, \mu_{z}\right)=1.52 \times 10^{3}\left(\mathrm{~kJ} / \mathrm{hr}^{2} \mathrm{~m}^{2}-\mathrm{K}\right)
$$

The $95 \%$ confidence region is then given by

$$
-758 \leq f(x, y, z) \leq 3.80 \times 10^{3}\left(\mathrm{~kJ} / \mathrm{hr}^{-\mathrm{m}^{2}}-\mathrm{K}\right)
$$

Since the value of the convective heat transfer coefficient cannot be negative, the $95 \%$ confidence region can be re-evaluated as

$$
0 \leq f(x, y, z) \leq 3.80 \times 10^{3}\left(\mathrm{~kJ} / \mathrm{hr}^{-\mathrm{m}^{2}}-\mathrm{K}\right)
$$

The nominal value of the convective heat transfer coefficient is

$$
f\left(\mu_{x}, \mu_{y}, \mu_{z}\right)=952\left(\mathrm{~kJ} / \mathrm{hr}^{\left.-\mathrm{m}^{2}-\mathrm{K}\right)}\right.
$$

The $95 \%$ confidence region obtained from the least-squares approach is compared to the $95 \%$ confidence regions obtained by the piece-wise approach and by Taylor series approximation, and the comparison is presented in Table 3.

The comparison of the results in Table 3 shows that the confidence interval of the convective heat transfer coefficient $(h)$ for the least-squares approach provides a better approximation to the more realistic (but much more computationally intensive) piecewise approach than the Taylor series approach. Furthermore, the narrow uncertainty

Table 3 A comparison of the $95 \%$ confidence interval

(Unit: $\mathrm{kJ} / \mathrm{hr}-\mathrm{m}^{2}-{ }^{\circ} \mathrm{K}$ )

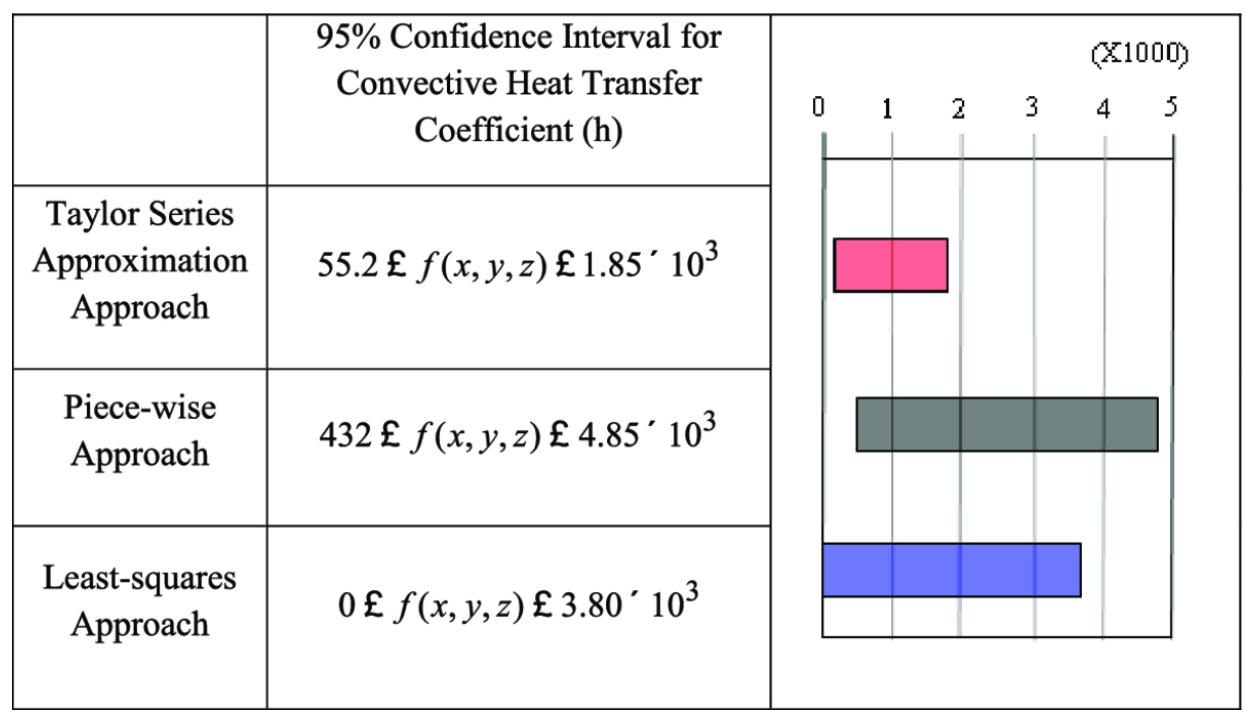


interval produced by the Taylor series approximation can lead to a "false sense of security" regarding the numerical value of the heat transfer coefficient.

\section{Conclusions}

A least-squares approach to linear uncertainty analysis has been described and illustrated. This approach can provide improved results over ordinary uncertainty propagation using a first order Taylor series approximation by minimizing the truncation errors in the linear approximation of the equation being analyzed. A drawback of this approach is that there is no explicit formula to find the sensitivity coefficients. However, in many instances the sensitivity coefficients are obtained through numerical derivatives. In such cases, there is little or no additional computational effort in obtaining the least-squares solution. This paper also shows a simple way to obtain the covariance matrix used in the uncertainty analysis. In many engineering applications, it is cumbersome to determine the correlation coefficients of the bias errors $(\rho)$, i.e., reasonable engineering judgment is required. Therefore, the authors recommend using covariance matrix expressed in terms of uncorrelated bias errors as shown in the third example. The results in the examples illustrate the advantages of using the least-squares approach.

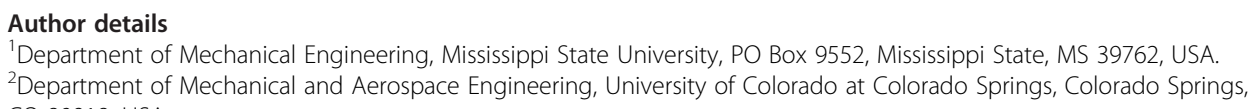

